\section{Evaluación de los programas de atención a la salud de las mujeres en las principales instituciones del sistema de salud de México}

\author{
Evaluation of women's health care programs in the \\ main institutions of the Mexican health system
}

\author{
Avaliação dos programas de atenção à saúde das \\ mulheres nas principais instituições do sistema de \\ saúde no México
}

\author{
1 Centro de Investigaciones \\ y Estudios Superiores en \\ Antropología Social, San \\ Cristóbal de Las Casas, \\ México. \\ 2 Instituto Nacional de Salud \\ Pública, San Cristóbal de Las \\ Casas, México. \\ Correspondencia \\ S. M. Navarro \\ Instituto Nacional de Salud \\ Pública. \\ Segunda Cerrada de San \\ Nicolás 6, Rancho San \\ Nicolás, San Cristóbal de \\ Las Casas, Chiapas 29240, \\ México. \\ sergio.meneses@insp.mx
}

\begin{abstract}
The aim of this study was to analyze the institutional capacity for provision of women's health care services in Mexico in accordance with prevailing regulations. A probabilistic national sample of health care institutions was used to compare performance rates according to services packages based on analysis of variance. No package showed outstanding performance. Adequate performance was seen in referral and counter-referral centers for uterine cervical cancer, childbirth care, breast cancer diagnosis, family planning counseling, and training in sexual and reproductive health. The lowest performance was seen in the prevention of uterine cervical cancer, obstetric urgencies, family and sexual violence, and promotion of family planning. All the institutions showed low performance in the prevention of breast cancer, promotion of family planning, and management of family and gender violence. The Ministry of Health's leadership needs to be strengthened in order to overcome resistance for the institutions to adhere to the prevailing regulations.
\end{abstract}

Program Evaluation; Women's Health; Health Systems
Graciela Freyermuth Enciso 1 Sergio Meneses Navarro 2 Martín Romero Martínez ${ }^{2}$

\section{Resumen}

El objetivo fue analizar la capacidad institucional de producción de servicios dirigidos a mujeres apegada a la normatividad. En una muestra probabilística nacional de las instituciones de salud, se compararon los índices de desempeño por paquetes de intervención mediante análisis de varianza. Ningún paquete tuvo un desempeño destacado. Con desempeño adecuado fueron los de referenciacontrarreferencia de cáncer cervicouterino, atención del parto, diagnóstico de cáncer de mama, consejería en planificación familiar y capacitación en salud sexual y reproductiva; los desempeños más bajos fueron los de prevención de cáncer cervicouterino, atención de las urgencias obstétricas, violencia familiar y sexual, y promoción de la planificación familiar. Todas las instituciones tienen un desempeño bajo en prevención de cáncer de mama, promoción de planificación familiar y atención a la violencia familiar y de género. Se debe fortalecer la función rectora de la Secretaría de Salud y vencer las resistencias para que las instituciones se apeguen a la normatividad.

Evaluación de Programas y Projectos de Salud; Salud de la Mujer; Sistemas de Salud 


\section{Introducción}

En el año 2012, a casi una década de la reforma que planteó extender la protección social en salud para todos, la Secretaría de Salud de México (SSA) declaró la cobertura universal en la materia 1 . No obstante, el sistema de salud continúa segmentado en instituciones que financian y prestan servicios para distintos sectores poblacionales -heterogéneos en sus características demográficas, perfil epidemiológico, gestión de riesgos y condiciones económicas-, al tiempo que existe cierta fragmentación operativa en virtud de la configuración descentralizada y la enorme extensión del sistema ${ }^{2}$. Esto ha contribuido a perpetuar y profundizar inequidades en el acceso, utilización, calidad y resultados en salud, dependiendo de en cuál institución, nivel de atención y entidad federativa se prestan los servicios 3,4. Para superar estos efectos negativos se ha planteado la integración funcional del sistema de salud 5,6,7. De acuerdo con este planteamiento, el sistema de salud debería desempeñar sus funciones de rectoría, prestación de servicios, financiamiento e inversión de manera integral, como si se tratase de un solo sistema y así los costos, condiciones de acceso y calidad de los servicios serían más homogéneos, apegados a una misma normatividad, mejorando la equidad 8,9.

La rectoría efectiva es fundamental para procurar que el acceso y provisión de servicios sean equitativos y con calidad. La Ley General de Salud confiere esta función a la SSA, si bien delega parte de la misma a los Servicios Estatales de Salud (SESA) y a las instituciones de la seguridad social 10. Se han realizado diversos esfuerzos por fortalecer la función rectora de la SSA. Uno de ellos ha sido la expedición de las Normas Oficiales Mexicanas (NOM) que proporcionan lineamientos técnicos obligatorios para todas las instituciones que prestan servicios de salud, intentando así homogeneizar su contenido y calidad. Por otro lado, se cuenta con los Programas de Acción Específicos (PAE) que responden a las prioridades definidas en los planes nacionales de desarrollo del poder ejecutivo federal (Subsecretaría de Prevención y Promoción de la Salud, Secretaría de Salud de México. Programas de acción específicos 2007-2012. http://www.spps. gob.mx/programas-y-proyectos.html, accedido el 11/Sep/2012). Adicionalmente, las Guías de Práctica Clínica (GPC) establecen referentes para la toma de decisiones clínicas y gerenciales (Centro Nacional de Excelencia Tecnológica en Salud, Secretaría de Salud de México. Nuevo catálogo maestro de guías de práctica clínica. http:/ /www. cenetec.salud.gob. $\mathrm{mx} /$ interior/catalogoMaestroGPC.html, accedido el 11/Sep/2012). Estos instrumentos son elaborados por consenso de expertos de las distintas instituciones de salud, basados en las mejores prácticas y evidencias científicas disponibles.

Con este marco se planteó el proyecto $\mathrm{Mo}$ nitoreo de la Atención a las Mujeres en Servicios Públicos del Sector Salud 11, con el objetivo de evaluar el desempeño del sistema de salud en su capacidad de producir servicios dirigidos a las mujeres en las principales instituciones públicas de salud del país, en alineación con lo establecido por la SSA en sus documentos rectores. Se usó a los PAE como los documentos rectores básicos, complementándolos con las NOM y GPC correspondientes. Se evaluaron las principales instituciones de seguridad social-Instituto Mexicano del Seguro Social (IMSS) e Instituto de Seguridad y Servicios Sociales de los Trabajadores del Estado (ISSSTE) - y las dirigidas a la antes denominada población abierta, ahora afiliada al Seguro Popular (SP) -SESA e IMSS-Oportunidades (IMSS-O). Esta evaluación es relevante porque permite hacer, por primera vez, una comparación del desempeño entre cuatro de las principales instituciones del sistema de salud -IMSS, IMSS-O, ISSSTE y SESA- con distintas características de operación, y que suelen ser evaluadas bajo sus propias lógicas institucionales.

\section{Metodología}

Se diseñó un monitoreo transversal que utilizó métodos cuantitativos. Se analizaron los siguientes programas: atención materna y perinatal 12, planificación familiar 13, salud sexual y reproductiva para adolescentes 14 , cáncer cervicouterino 15 , cáncer de mama 16 y prevención y atención de la violencia familiar y de género ${ }^{17}$. Con base en los lineamientos de los PAE, las NOM y las GPC se definieron paquetes de intervenciones, constituidos por el conjunto de intervenciones mínimas indispensables que hacen posible la producción adecuada del servicio 18. Se definieron 620 intervenciones integradas en 25 paquetes para el análisis de los seis PAE con sus respectivos índices de desempeño global.

Para la recolección de información se diseñaron cuatro instrumentos: (1) guías de cotejo, (2) cuestionarios, (3) guías de observación y (4) guías de entrevista para diversos actores. Se definieron 19 ámbitos de recolección de información con informantes específicos en cada establecimiento, según nivel de atención. Estos instrumentos fueron consensados con los responsables de los programas en cada institución.

Las unidades médicas fueron seleccionadas mediante muestreo estratificado y de conglome- 
rados. Se definieron cinco regiones y en cada una se seleccionaron diez municipios, de acuerdo con la probabilidad proporcional a su población. Se establecieron dos criterios de estratificación en cada municipio: la institución (SSA, IMSS, ISSSTE, IMSS-O) y el tipo de establecimiento (consulta externa y hospitalización) (Tabla 1). Luego, a cada unidad en muestra se le asignó un ponderador $w$ que depende de la probabilidad de selección. El marco de muestreo empleado fue el directorio de establecimientos médicos a nivel nacional del sector salud, publicado por la SSA en la página electrónica: http://clues.salud. gob.mx/. La muestra se conformó por 205 unidades médicas, en 50 municipios de 24 entidades federativas, de las cuales se obtuvo respuesta en 201 unidades ( $2 \%$ de no respuesta). El número de muestra se definió a partir de un estudio de desempeño de servicios maternos; por ello la principal debilidad del estudió radicó en el número de muestra que fue insuficiente para evaluar algunos de los paquetes, en especial del programas de salud sexual y reproductiva para adolescentes. La representatividad es nacional en función de las instituciones que prestan los servicios y del tipo de población a la que atienden.

El trabajo de campo se llevó a cabo de septiembre a diciembre de 2010 en 201 unidades (no se obtuvo información en cuatro unidades), teniendo una tasa de respuesta de $98 \%$. Para la captura de información se usó el software CSPro Census and Survey Processing System (http:// www.census.gov/population/international/ software/cspro/). Para la evaluación de los diferentes paquetes de intervención se utilizaron índices que representan una calificación relativizada con respecto al total de ítems evaluados de acuerdo con los servicios que otorgaba cada unidad 11 , y se calcula como el cociente de la suma de puntos acumulados por una unidad médica, dividido entre el número de ítems evaluados en esa unidad, el valor mínimo del puede ser de 0 y el máximo 1. Se realizó una cromatización de los índices utilizando la escala de valoración de desempeño propuesta por el Consejo Nacional de Evaluación de la Política Social (CONEVAL): desempeño destacado de 0.85 o mayor, desempeño adecuado de 0,70 a 0,84 , desempeño moderado de 0,50 a 0,69 , bajo desempeño menor a 0,50 . Las pruebas de significancia se realizaron para comparar los índices obtenidos entre las instituciones y niveles de atención. Se corrieron todas las ANOVA, pero solamente se considerarán en la descripción el valor $\mathrm{p} \leq 0,01$ y $\mathrm{p} \leq 0,05$. Esta investigación fue aprobada por Comité de Ética de Investigación del ISSSTE y se contó con el consentimiento escrito de cada institución y de los participantes para llevar a cabo la evaluación, además de preservar la confidencialidad de los informantes.

\section{Resultados}

\section{Desempeño global de los programas de acción específicos}

El programa que tuvo mejor desempeño, a juzgar por el índice global obtenido, fue el de cáncer cervicouterino con un índice de 0,68 , seguido por el de planificación familiar con 0,62 , salud sexual y reproductiva para adolescentes con 0,61 , cáncer de mama con 0,60 , atención materna y perinatal con $0,56 \mathrm{y}$, finalmente, el de prevención y atención de la violencia familiar y de género con 0,46 . Todos los programas fueron calificados con desempeño moderado, salvo el de prevención y atención de la violencia familiar y de género que

Establecimientos médicos por tipo de unidad e institución en la muestra. México, 2010.

\begin{tabular}{lccc}
\hline Institución & Consulta externa & Hospitalización & Total \\
\hline SSA & 49 & 34 & 83 \\
IMSS & 35 & 24 & 59 \\
IMSS-O & 13 & 6 & 19 \\
ISSSTE & 21 & 19 & 40 \\
Total & 118 & 83 & 201 *
\end{tabular}

IMSS: Instituto Mexicano del Seguro Social; IMSS-O: Instituto Mexicano del Seguro Social-Oportunidades; ISSSTE: Instituto de Seguridad y Servicios Sociales de los Trabajadores del Estado; SSA: Secretaría de Salud de México.

* El total de la muestra fue de 205, en 4 unidades no se obtuvo respuesta.

Fuente: Freyermuth et al. 11 
Tabla 2

Índices de desempeño por programa, instituciones y nivel de atención. México, 2010.

\begin{tabular}{|c|c|c|c|c|c|c|c|c|c|}
\hline PAE & $\begin{array}{l}\text { Índice } \\
\text { SESA }\end{array}$ & $\begin{array}{l}\text { Índice } \\
\text { IMSS }\end{array}$ & $\begin{array}{l}\text { Índice } \\
\text { ISSSTE }\end{array}$ & $\begin{array}{c}\text { Índice } \\
\text { IMSS-O }\end{array}$ & $\begin{array}{c}\text { Índice } \\
\text { global por } \\
\text { paquete }\end{array}$ & $\begin{array}{l}\text { Índice } \\
\text { primer } \\
\text { nivel }\end{array}$ & $\begin{array}{c}\text { Índice } \\
\text { segundo } \\
\text { nivel }\end{array}$ & $\begin{array}{c}\text { Índice de } \\
\text { instituciones } \\
\text { que atienden } \\
\text { a población } \\
\text { abierta y } \\
\text { del SP } \\
\text { (SESA/IMSS-O) }\end{array}$ & $\begin{array}{c}\text { Índice de } \\
\text { instituciones } \\
\text { que atienden } \\
\text { a población } \\
\text { asegurada } \\
\text { (IMSS/ISSSTE) }\end{array}$ \\
\hline
\end{tabular}

\begin{tabular}{|c|c|c|c|c|c|c|c|c|c|}
\hline $\begin{array}{l}\text { Atención materna y } \\
\text { perinatal }\end{array}$ & 0,56 * & 0,57 & 0,56 & 0,54 * & 0,56 & 0,55 ** & $0,68 * *$ & 0,56 & 0,60 \\
\hline Cáncer cervicouterino & 0,66 & 0,78 ** & 0,60 & 0,79 ** & 0,68 & 0,68 & 0,68 & 0,67 & 0,73 \\
\hline Cáncer de mama & 0,62 & 0,58 & 0,53 & 0,52 & 0,60 & 0,61 & 0,55 & 0,61 & 0,57 \\
\hline Planificación familiar & 0,62 * & 0,64 * & 0,50 & 0,77 ** & 0,62 & 0,62 & 0,58 & 0,62 & 0,60 \\
\hline $\begin{array}{l}\text { Salud sexual y } \\
\text { reproductiva para } \\
\text { adolescentes }\end{array}$ & 0,60 & 0,63 & 0,51 & 0,63 & 0,61 & 0,61 & & 0,61 & 0,61 \\
\hline $\begin{array}{l}\text { Prevención y atención } \\
\text { de la violencia familiar y } \\
\text { de género }\end{array}$ & 0,49 & $0,35 * \star$ & 0,32 ** & 0,40 * & 0,46 & 0,46 & 0,46 & 0,48 ** & 0,34 ** \\
\hline
\end{tabular}

IMSS: Instituto Mexicano del Seguro Social; IMSS-O: Instituto Mexicano del Seguro Social-Oportunidades; ISSSTE: Instituto de Seguridad y Servicios Sociales de los Trabajadores del Estado; PAE: Programas de Acción Específicos; SESA: Servicios Estatales de Salud; SP: Seguro Popular.

${ }^{*} \mathrm{p}<0,05$

** $p>0,01$.

Nota: en la cromatización el desempeño destacado corresponde a > 0,85 (color blanco), desempeño adecuado 0,70-0,84 (tono gris claro), desempeño moderado 0,50-0,69 (tono gris intermedio) y bajo desempeño < 0,50 (tono gris más oscuro).

Fuente: elaboración propia con datos de la encuesta.

tuvo desempeño bajo (Tabla 2). En cuanto a su desempeño según nivel de atención, solamente se encontró diferencia significativa en el programa atención materna y perinatal con mejor desempeño en el segundo nivel. El programa prevención y atención de la violencia familiar y de género presentó también diferencia significativa en su desempeño por tipo de población, con mejor desempeño en la población que acude a los SESA-IMSS-O y el programa atención materna y perinatal con mejor desempeño para población asegurada (IMSS/ISSSTE). Los programas prevención y atención de la violencia familiar y de género, planificación familiar y cáncer cervicouterino tuvieron diferencias en su desempeño entre instituciones. En el caso de prevención y atención de la violencia familiar y de género, los SESA presentaron mejor desempeño con respecto al de las instituciones, en planificación familiar se presentaron diferencias significativas en el menor desempeño del ISSSTE con respecto al resto de las instituciones y en el cáncer cervicouterino en el mejor desempeño en el IMSS e IMSS-O en relación con los SESA e ISSSTE (Tabla 2).

\section{Desempeño general de los paquetes de intervención}

En general, ninguno de los 25 paquetes de intervención tuvo desempeño destacado. Fueron calificados como de desempeño adecuado los paquetes de referencia y contrarreferencia de cáncer cervicouterino, atención del parto, diagnóstico de cáncer de mama, capacitación en SSRA y consejería en planificación familiar; con desempeño moderado se encontraron los paquetes de disponibilidad de métodos de planificación familiar, diagnóstico para tratamiento oportuno de cáncer cervicouterino, atención prenatal, anticoncepción post evento obstétrico, atención del recién nacido, consejería en salud sexual y reproductiva para adolescentes, detección de cáncer de mama, detección de virus de inmunodeficiencia humana (VIH) durante el embarazo, prevención y tratamiento de cáncer de mama, accesibilidad a servicios de salud sexual y reproductiva para adolescentes y detección y atención de influenza durante el embarazo. Los paquetes de intervención calificados con bajo desempe- 
ño son: prevención de cáncer cervicouterino, accesibilidad a servicios de atención materna y perinatal, detección de sífilis e infecciones de transmisión sexual (ITS) durante el embarazo, violencia sexual, atención de las emergencias obstétricas, violencia familiar y promoción de la planificación familiar (Tabla 3).

Desempeño de los paquetes de intervención por institución

En el desempeño por paquetes de intervención y tipo de institución destacan los siguientes resultados (Tabla 3): todas las instituciones tienen bajo desempeño en detección de VIH durante el embarazo, salvo los SESA que tienen desempeño moderado $(0,63)$; las instituciones de seguridad social brindan desempeño moderado para la atención de las emergencias obstétricas, mientras que los SESA e IMSS-O tienen bajo desempeño en la materia; además, la atención de urgencias obstétricas tiene mejor desempeño en el segundo nivel de atención; en general, el IMSS e IMSS-O tienen mejor desempeño (adecuado) en los paquetes referidos al programa cáncer cervicouterino, siendo este programa el de mejor desempeño para esta institución; todas las instituciones tienen bajo desempeño en prevención de cáncer de mama, salvo los SESA con desempeño moderado y todas se encuentran en la medianía, en cuanto a actividades de detección oportuna sin diferencias entre ellas; la promoción de la planificación familiar se encuentra con bajo desempeño en todas las instituciones salvo en el IMSS-O, y sólo el IMSS e IMSS-O presentan una adecuada disponibilidad de métodos; respecto al programa prevención y atención de la violencia familiar y de género todas las instituciones tienen bajo desempeño, si bien la prevención de la violencia familiar es mejor en instituciones que atienden a población abierta o afiliada al SP, y la prevención de la violencia sexual en el segundo nivel de atención.

El IMSS-O tuvo mejor desempeño estadísticamente significativo en el paquete de prevención de cáncer cervicouterino y promoción de la planificación familiar. Los SESA mostraron mejor desempeño en prevención de cáncer de mama, detección de VIH y prevención de la violencia familiar. El IMSS mostró mejor desempeño frente a los SESA en atención de las emergencias obstétricas, detección de sífilis e ITS y accesibilidad del programa atención materna y perinatal. El paquete de diagnóstico de cáncer cervicouterino mostró mejor desempeño en el IMSS e IMSS-O.

\section{Discusión}

En materia de salud de las mujeres, México enfrenta un panorama epidemiológico complejo de doble carga de enfermedad. Por un lado, aún afronta añejos problemas asociados con la reproducción como elevadas razones de morbi-mortalidad materna ${ }^{19}$ y por cáncer cervicouterino 19,20 , elevadas tasas de natalidad 21 e insuficiente cobertura de métodos de planificación familiar 21, además del profundo fenómeno de violencia doméstica y de género 22,23,24. Por otro lado, encara problemas emergentes, costosos y de mayor complejidad que contribuyen crecientemente con una mayor carga de enfermedad y muerte, como el cáncer de mama 25 o la morbi-mortalidad por influenza durante embarazo 26,27.

Los hallazgos del presente monitoreo resultan pertinentes para analizar la respuesta que implementa el sistema de salud para enfrentar este difícil contexto, sobre todo en las capacidad de producción de servicios y su rectoría en las diversas instituciones del sector público. Ninguno de los programas analizados tuvo óptimo desempeño global que garantice la producción de servicios en apego pleno a la normatividad vigente que les rige. Por el contrario, la tendencia es hacia el bajo o moderado desempeño global en estos seis programas prioritarios.

Enfocarse en los paquetes de intervención permite un análisis comparativo más detallado, destacándose algunos resultados preocupantes, dado su bajo desempeño: prevención de cáncer cervicouterino (que se evaluó a través de la disponibilidad y aplicación de la vacuna contra el virus del papiloma humano -VPH-), prevención y detección de cáncer de mama, promoción de la planificación familiar, atención de las emergencias obstétricas y atención a la violencia familiar y de género.

El monitoreo identifica un desempeño moderado para los paquetes de diagnóstico oportuno de cáncer cervicouterino en el primero y segundo niveles de atención para los SESA e ISSSTE, si bien en el caso del IMSS ordinario y oportunidades el desempeño es adecuado (pero no destacado). Cabe señalar que en las actividades de tamizaje para la detección de cáncer cervicouterino, únicamente el IMSS-O se basa en la inspección visual con ácido acético del cuello del útero -de alta sensibilidad pero menor especificidad para la detección ${ }^{28-}$ y no en la prueba de Papanicolaou como lo norman el PAE 15, la NOM y la GPC respectivas. Dada la importancia del diagnóstico oportuno de este tipo de cáncer y del tipo de población que atiende el IMSS-O (prioritariamente pobre e indígena), consideramos que se debe destacar este hallazgo. El cervicouterino 
Tabla 3

Paquetes de intervención por programa e índices de desempeño global, por nivel de atención e institución. México, 2010.

\begin{tabular}{|c|c|c|c|c|c|c|c|c|c|}
\hline $\begin{array}{l}\text { PAE/Paquetes de } \\
\text { intervención por PAE }\end{array}$ & $\begin{array}{l}\text { Índice } \\
\text { SESA }\end{array}$ & $\begin{array}{l}\text { Índice } \\
\text { IMSS }\end{array}$ & $\begin{array}{l}\text { Índice } \\
\text { ISSSTE }\end{array}$ & $\begin{array}{l}\text { Índice } \\
\text { IMSS-O }\end{array}$ & $\begin{array}{c}\text { Índice } \\
\text { global por } \\
\text { paquete }\end{array}$ & $\begin{array}{c}\text { Índice } \\
\text { primer } \\
\text { nivel }\end{array}$ & $\begin{array}{c}\text { Índice } \\
\text { segundo } \\
\text { nivel }\end{array}$ & $\begin{array}{c}\text { Índice de } \\
\text { instituciones } \\
\text { que atienden } \\
\text { a población } \\
\text { abierta y } \\
\text { del SP }\end{array}$ & $\begin{array}{c}\text { Índice de } \\
\text { instituciones } \\
\text { que atienden } \\
\text { a población } \\
\text { asegurada }\end{array}$ \\
\hline
\end{tabular}

\begin{tabular}{|c|c|c|c|c|c|c|c|c|c|}
\hline \multicolumn{10}{|c|}{ Atención materna y perinatal } \\
\hline $\begin{array}{l}\text { Accesibilidad para la } \\
\text { población }\end{array}$ & 0,47 & 0,57 & 0,53 & 0,53 & 0,49 & 0,48 & 0,51 & 0,47 & 0,56 \\
\hline Atención prenatal & 0,69 & 0,66 & 0,63 & 0,51 & 0,67 & 0,67 & 0,72 & 0,67 & 0,65 \\
\hline Sífilis e ITS & 0,46 & 0,51 & 0,55 & 0,51 & 0,47 & 0,46 & 0,66 & 0,46 & 0,52 \\
\hline VIH & 0,63 & 0,42 & 0,37 & 0,36 & 0,58 & 0,59 & 0,44 & 0,61 & 0,41 \\
\hline Influenza & 0,49 & 0,62 & 0,47 & 0,58 & 0,51 & 0,50 & 0,63 & 0,50 & 0,58 \\
\hline Atención del parto & 0,76 & 0,71 & 0,69 & 0,76 & 0,74 & 0,76 & 0,73 & 0,76 & 0,71 \\
\hline $\begin{array}{l}\text { Atención del recién } \\
\text { nacido }\end{array}$ & 0,63 & 0,69 & 0,60 & 0,68 & 0,65 & 0,56 & 0,73 & 0,64 & 0,66 \\
\hline $\begin{array}{l}\text { Atención de la } \\
\text { emergencia obstétrica }\end{array}$ & 0,45 & 0,58 & 0,55 & 0,49 & 0,47 & 0,45 & 0,71 & 0,45 & 0,57 \\
\hline \multicolumn{10}{|l|}{ Cáncer cervicouterino } \\
\hline Prevención & 0,25 & 0,71 & 0,37 & 0,81 & 0,34 & 0,33 & 0,46 & 0,29 & 0,62 \\
\hline $\begin{array}{l}\text { Diagnóstico consulta } \\
\text { externa }\end{array}$ & 0,66 & 0,78 & 0,60 & 0,79 & 0,67 & 0,68 & 0,68 & 0,67 & 0,73 \\
\hline $\begin{array}{l}\text { Referencia y contra- } \\
\text { referencia }\end{array}$ & 0,76 & 0,75 & 0,68 & 0,74 & 0,75 & 0,76 & 0,68 & 0,75 & 0,74 \\
\hline $\begin{array}{l}\text { Diagnóstico para } \\
\text { tratamiento oportuno, } \\
\text { segundo nivel }\end{array}$ & 0,65 & 0,81 & 0,56 & 0,82 & 0,67 & 0,67 & & 0,66 & 0,74 \\
\hline \multicolumn{10}{|l|}{ Cáncer de mama } \\
\hline Prevención & 0,60 & 0,47 & 0,36 & 0,45 & 0,56 & 0,57 & 0,45 & 0,59 & 0,44 \\
\hline Detección & 0,63 & 0,63 & 0,59 & 0,65 & 0,63 & 0,62 & 0,68 & 0,63 & 0,62 \\
\hline Diagnóstico & 0,73 & 0,80 & 0,84 & 0,77 & 0,77 & - & 0,77 * & 0,75 * & 0,81 * \\
\hline Tratamiento & 0,52 & 0,56 & 0,66 & 0,42 & 0,52 & - & 0,52 & 0,49 & 0,59 \\
\hline \multicolumn{10}{|l|}{ Planificación familiar } \\
\hline Promoción & 0,36 & 0,25 & 0,19 & 0,54 & 0,35 & 0,36 & 0,30 & 0,37 & 0,24 \\
\hline Consejería & 0,74 & 0,71 & 0,60 & 0,68 & 0,73 & 0,73 & 0,68 & 0,73 & 0,68 \\
\hline Disponibilidad & 0,67 & 0,78 & 0,57 & 0,82 & 0,69 & 0,68 & 0,75 & 0,68 & 0,72 \\
\hline $\begin{array}{l}\text { Anticoncepción post } \\
\text { evento obstétrico }\end{array}$ & 0,58 & 0,75 & 0,46 & 0,77 & 0,66 & - & 0,66 & 0,65 & 0,67 * \\
\hline \multicolumn{10}{|l|}{$\begin{array}{l}\text { Salud sexual y reproductiva } \\
\text { para adolescentes }\end{array}$} \\
\hline Accesibilidad & 0,51 & 0,51 & 0,44 & 0,61 & 0,52 & 0,52 & - & 0,52 & 0,50 \\
\hline Consejería & 0,65 & 0,68 & 0,56 & 0,58 & 0,64 & 0,64 & - & 0,64 & 0,66 \\
\hline Capacitación & 0,60 & 0,67 & 0,48 & 0,71 & 0,62 & 0,62 * & - & 0,61 * & 0,63 * \\
\hline
\end{tabular}

(continúa) 
Tabla 3 (continuación)

\begin{tabular}{|c|c|c|c|c|c|c|c|c|c|}
\hline $\begin{array}{l}\mathrm{PAE} / \text { Paquetes de } \\
\text { intervención por PAE }\end{array}$ & $\begin{array}{l}\text { Índice } \\
\text { SESA }\end{array}$ & $\begin{array}{l}\text { Índice } \\
\text { IMSS }\end{array}$ & $\begin{array}{l}\text { Índice } \\
\text { ISSSTE }\end{array}$ & $\begin{array}{l}\text { Índice } \\
\text { IMSS-O }\end{array}$ & $\begin{array}{c}\text { Índice } \\
\text { global por } \\
\text { paquete }\end{array}$ & $\begin{array}{c}\text { Índice } \\
\text { primer } \\
\text { nivel }\end{array}$ & $\begin{array}{c}\text { Índice } \\
\text { segundo } \\
\text { nivel }\end{array}$ & $\begin{array}{c}\text { Índice de } \\
\text { instituciones } \\
\text { que atienden } \\
\text { a población } \\
\text { abierta y } \\
\text { del SP }\end{array}$ & $\begin{array}{c}\text { Índice de } \\
\text { instituciones } \\
\text { que atienden } \\
\text { a población } \\
\text { asegurada }\end{array}$ \\
\hline
\end{tabular}

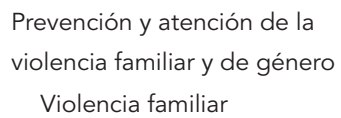

IMSS: Instituto Mexicano del Seguro Social; IMSS-O: Instituto Mexicano del Seguro Social-Oportunidades; ISSSTE: Instituto de Seguridad y Servicios Sociales de los Trabajadores del Estado; ITS infecciones de transmisión sexual (ITS); PAE: Programas de Acción Específicos; SESA: Servicios Estatales de Salud; SP: Seguro Popular.

es la segunda principal causa de muerte por cáncer en mujeres mayores de 25 años del país 29. Este dato evidencia un tropiezo del programa de detección oportuna, toda vez que se trata de una enfermedad prevenible y curable cuando se detecta de manera temprana. La Encuesta Nacional de Salud y Nutrición 2012 (ENSANUT) coincide con los resultados del presente monitoreo. Esta encuesta revela, además, que la cobertura del examen de Papanicolaou en mujeres de 25 a 64 años es menor entre la población no asegurada, en condiciones de pobreza multidimensional y en el quintil de ingresos inferior 20,30. Así, el mayor esfuerzo para incrementar la oferta y demanda de la detección oportuna mediante Papanicolaou y detección de VPH debe focalizarse a estos sectores poblacionales. Además, sería apropiado especificar en los documentos rectores la pertinencia e indicaciones del uso de la inspección visual con ácido acético como técnica de tamizaje.

Si bien desde 2006 el cáncer de mama representa la principal causa de muerte por cáncer en mujeres mayores de 25 años en México, los resultados del monitoreo señalan que aún falta mucho para fortalecer las actividades de información para la prevención y detección oportuna de esta enfermedad. Los paquetes de intervención en esta materia tuvieron un desempeño de moderado a bajo en todas las instituciones y el hallazgo toma mayor relevancia, si se considera que la mayoría de los casos se detectan en etapas avanzadas del padecimiento. Las autoridades de salud reconocen que tanto la oferta, como la demanda de servicios de detección temprana, son escasas e insuficientes, sobre todo entre las poblaciones más pobres, no afiliadas a la seguridad social y en las entidades del sureste del apaís 20,25,30. En este contexto, el hecho de que no se difundan las limitadas medidas preventivas conocidas y, sobre todo, no se realice la detección oportuna, contribuye a ahondar y prolongar este apremiante problema y sus profundas consecuencias sociales y económicas, tanto para la población, como para el sistema de salud 25.

La promoción de la planificación familiar tuvo bajo desempeño en todas las instituciones y sólo el IMSS e IMSS-O presentan una adecuada disponibilidad de métodos. La planificación familiar, además de un derecho constitucional 31, es una de las principales intervenciones para disminuir la morbi-mortalidad materna ${ }^{32}$. Así, la insuficiente promoción y disponibilidad de métodos de planificación familiar, no sólo se contraponen al mencionado precepto constitucional, sino que no contribuyen a la meta de disminuir la mortalidad materna de conformidad con los Objetivos de Desarrollo del Milenio 33. Los tomadores de decisiones del sistema de salud tienen el reto de superar estas debilidades en la implementación del programa de planificación familiar.

Desde la década de 1990, se reconoce que aproximadamente $15 \%$ de las mujeres presentará una complicación aguda e inesperada relacionada con la maternidad, que pondrá en riesgo su vida 34 . Afortunadamente, contamos con los conocimientos y tecnologías médicas que permiten enfrentar con éxito la mayoría de estas complicaciones y, si son implementadas oportunamente, evitar el deceso. Este recono- 
cimiento ha llevado a un cambio de paradigma que coloca a los servicios de atención de las emergencias obstétricas como uno de los pilares de la política contra la muerte materna 35 . Desde el año 2008, México incorporó este enfoque en el programa atención materna y perinatal 12 , y en 2010 lo corroboró con la firma del Convenio General de Colaboración Interinstitucional para la Atención de la Emergencia Obstétrica, el mismo que pretende facilitar el acceso oportuno y sin costo a servicios de atención de las emergencias obstétricas a toda mujer con alguna complicación que ponga en riesgo su vida, en cualquier establecimiento de la SSA, los SESA, IMSS o ISSSTE, sin importar su condición de afiliación, capacidad de pago o distingo de cualquier índole 36,37 . No obstante, los resultados del monitoreo muestran un preocupante bajo desempeño en la capacidad de producir servicios de atención de las emergencias obstétricas, sobre todo en las instituciones dirigidas a la población sin seguridad social. El IMSS y el ISSSTE tuvieron desempeño mediano que tampoco es satisfactorio y es incongruente con el propósito de disminuir la muerte materna.

El programa prevención y atención de la violencia familiar y de género fue el que tuvo el desempeño más bajo en todas las instituciones. Este es un aspecto relevante, puesto que evidencia que, más allá del discurso político, de hecho las instituciones de salud aún no logran instrumentar una respuesta eficiente y efectiva para la atención de este importante problema de salud pública.

Los Artículos $1^{\circ}$ y $4^{\circ}$ de la Constitución Política de los Estados Unidos Mexicanos establecen la igualdad ante la ley y el derecho a la protección de la salud de todos los ciudadanos 33 . En correspondencia, los PAE, las NOM y las GPC establecen los recursos, actividades y procesos para garantizar la calidad y homogeneidad de los servicios en cualquier institución donde se brinden, sin distinción de ninguna índole. No obstante, el presente monitoreo muestra que el desempeño del sistema de salud es heterogéneo entre instituciones y regiones, si bien ninguna institución presenta sistemáticamente mejores desempeños que otra. Esto tiene repercusiones respecto a la equidad, pues implica que se prestan servicios con diferente contenido y calidad a los distintos sectores poblacionales, de acuerdo con su afiliación institucional, lugar y causa de atención. Esto constituye un reto para la equidad y efectividad de la respuesta ante las dinámicas condiciones de salud, en un país donde las brechas en salud siguen ocupando un lugar preponderante 37,38 .

Por otro lado, los resultados del monitoreo también ponen de manifiesto algunos límites de la función rectora de la SSA y evidencian las resistencias de las instituciones de la seguridad social a someterse a dicha dirección. Sin embargo, para lograr un sistema de salud universal, integrado, eficiente y sostenible es necesario vencer dichas resistencias y fortalecer la función rectora de la SSA. Esta información relevante debe alertar a los tomadores de decisión para profundizar en el análisis de los determinantes del bajo desempeño de estos paquetes de intervención e implementar medidas correctivas urgentes.

\section{Conclusiones}

El desempeño de los programas analizados es insuficiente en general. Existen aspectos críticos que requieren atención urgente. Entre éstos destacan la promoción y detección de cáncer cervicouterino y cáncer de mama, la atención de las emergencias obstétricas, la promoción y disponibilidad de métodos de planificación familiar y el mejoramiento de la atención a la violencia familiar y de género en establecimientos de salud.

Si bien no hay una diferencia constante en la capacidad de producción de servicios entre instituciones para población abierta -o del SP versus los de población asegurada-, estos resultados nos muestran que hay una tendencia hacia una homogeneización a la baja en el desempeño de los servicios de salud. Aunque el Artículo $4^{\circ}$ de la Constitución señala que todos los mexicanos tienen el mismo derecho a la protección de la salud, en los hechos existe un trato diferenciado entre las personas con o sin seguridad social, según la causa, lugar y nivel de atención, debido en parte a que el sistema mexicano de salud se encuentra segmentado y fragmentado. En este contexto, la función rectora de la SSA enfrenta dificultades y retos. Cada institución del sistema de salud posee cierta autoridad para definir e implementar sus programas, decidir el manejo financiero o establecer mecanismos de coordinación, es decir, ejercer funciones de rectoría al interior de su organización. De este modo, la función rectora de la SSA enfrenta el enorme reto de integrar una respuesta coherente y eficiente entre las instituciones que componen el sistema de salud, y congruente y efectiva ante las necesidades de la población en general.

La segmentación actual del sistema de salud basado en clientelas conlleva inconvenientes en detrimento de su eficacia, eficiencia y equidad: permanecen las inequidades en el financiamiento y acceso a los servicios de salud entre afiliados y no afiliados de las distintas instituciones, se ensancha el aparato burocrático con el consecuente incremento de los costos y se duplican 
acciones de prestación, financiamiento, inversión y rectoría.

A partir del análisis de los programas producto del presente monitoreo, señalamos las siguientes recomendaciones para mejorar la respuesta a las necesidades de salud de las mujeres:

Proponer modificaciones estructurales en el financiamiento del sistema y vencer las resistencias para que todas las instituciones se apeguen a los PAE, NOM y GPC, para consolidar la función rectora de la SSA.

Es deseable contar con un fondo único, donde se mancomunen los recursos financieros del sistema de salud, y poseer un sistema de registro nominal único de afiliados a cualquier seguro médico público. Esto permitiría tener una idea precisa de las personas afiliadas a cada sub-sistema y realizar los pagos correspondientes con mayor eficiencia y justicia financiera 39 .

Crear redes de servicio interinstitucionales explícitas, señaladas con claridad en los modelos operativos de los PAE y Planes Estatales de Salud.

Desarrollar planes interinstitucionales de inversión y desarrollo de infraestructura y recursos para el primer y segundo niveles. Esto contribuiría a evitar duplicidades y a implementar una respuesta más eficiente ante problemas prioritarios como la detección oportuna de cáncer de mama o la disponibilidad de métodos de planificación familiar. Además, estos planes de inversión serían financiados a través del fondo único propuesto.

Fortalecer urgentemente las acciones para garantizar el acceso universal a la atención de las emergencias obstétricas, a la promoción y disponibilidad de métodos de planificación familiar, al tamizaje para la detección de cáncer cervicouterino, mediante Papanicolaou y prueba de VPH, a servicios de detección temprana de cáncer de mama e incorporar la atención efectiva a la violencia familiar y de género en establecimientos de salud.

Finalmente, es importante continuar con ejercicios de monitoreo independiente del desempeño de los programas de salud dirigidos a las mujeres, para evaluar el avance en la materia y tomar las medidas correctivas necesarias.

El presente monitoreo y las recomendaciones que surgen del mismo apuntan a fortalecer un sistema de salud centrado en las personas, eficiente, sostenible, con servicios de alta calidad, enfocado tanto en la prevención, como en la atención médica, sobre todo entre poblaciones más vulnerables y con una rectoría efectiva que haga realidad la meta del acceso universal a los servicios de salud para todas las mujeres del país.

\section{Resumo}

O objetivo foi analisar a capacidade institucional de produção de serviços dirigidos às mulheres, segundo os regulamentos. Numa amostra de probabilidades das instituições de saúde, em nível nacional, compararam-se os índices de desempenho por conjuntos de intervenção usando-se a análise de variantes. Nenhum conjunto teve um desempenho notório. Com desempenho adequado apresentaram-se os de referência-contrarreferência de cancro do colo do útero, assistência ao parto, diagnóstico de cancro de mama, aconselhamento no planejamento familiar e capacitação sobre saúde sexual e reprodutiva; os desempenhos mais baixos foram os de prevenção de cancro do colo do útero, assistência em urgências obstétricas, violência familiar e sexual, e promoção do planejamento familiar. Todas as instituições têm um baixo desempenho na prevenção de cancro de mama, na promoção do planejamento familiar e assistência nos casos de violência familiar e de gênero. É necessário fortalecer a função diretiva da Secretaria de Saúde e superar as resistências, para que as instituições se apeguem aos regulamentos.

Avaliação de Programas e Projetos de Saúde; Saúde da Mulher; Sistemas de Saúde

\section{Colaboradores}

G. F. Enciso guió el desarrollo de las actividades de recolección de datos, análisis e interpretación; fue responsable por la conceptualización del presente artículo, dirigió y participó en su redacción y aprobó la versión final. S. M. Navarro colaboró en el diseño conceptual y metodológico de algunos componentes del monitoreo, así como en el análisis e interpretación de los datos; participó sustancialmente en la conceptualización y redacción del presente artículo, así como en la aprobación de la versión final. M. R. Martínez colaboró en el diseño de la muestra del estudio, el análisis estadístico, revisión y retroalimentación de la versión preliminar y final del presente manuscrito.

\section{Agradecimientos}

Agradecemos a las autoridades de la Secretaría de Salud Federal, del IMSS, IMSS-Oportunidades, ISSSTE y de los Servicios Estatales de Salud por las facilidades brindadas para la realización del presente trabajo de evaluación. Al Instituto Nacional de las Mujeres por el financiamiento. 


\section{Referencias}

1. Knaul F, González-Pier E, Gómez-Dantés O, García-Junco D, Arreola-Ornelas H, Barraza-Lloréns $\mathrm{M}$, et al. The quest for universal health coverage: achieving social protection for all in Mexico. Lancet 2012; 380:1259-79.

2. Gómez-Dantés O, Sesma S, Becerril V, Knaul FM, Arreola-Ornelas H, Frenk J, et al. Sistema de salud de México. Salud Pública Méx 2011; 53 Suppl 2:S220-32.

3. Secretaría de Salud de México. Sistema de protección social en salud: elementos conceptuales, financieros y operativos. México DF: Secretaría de Salud de México; 2005.

4. Ares-Parga R. Propuesta de reforma estructural para el Sistema Nacional de Salud. Gac Méd Méx 2011; 147:475-81.

5. Londoño JL, Frenk J. Structured pluralism: towards an innovative model for health system reform in Latin America. Health Policy 1997; 41:1-36.

6. Soberón G, Valdéz C. Evidencias y salud. ¿̇Hacia dónde va el sistema de salud en México? Salud Pública Méx 2007; 49:5-27.

7. Presidencia de la República. Visión 2030: el México que queremos, Estados Unidos Mexicanos. http://www.vision2030.gob.mx/ (accedido el 11/ Sep/2012).

8. World Health Organization. Integrated health services: what and why? Making health systems Works. Geneva: World Health Organization; 2008. (Technical Brief, 1).

9. Grupo de Trabajo de la Fundación Mexicana para la Salud. Universalidad de los servicios de salud en México. Salud Pública Méx 2013; 55(n.spe):1-64.

10. Secretaría de Gobernación. Ley General de Salud. Diario Oficial de la Federación 2012; 7 jun.

11. Freyermuth G, Meléndez D, Argüello H, Montoya C, Bosch M, Reartes D, et al. Monitoreo a la atención de las mujeres en servicios públicos del sector salud. México DF: Instituto Nacional de las Mujeres; 2011. (Cuadernos de Trabajo, 29).

12. Secretaría de Salud de México. Programa de acción específico 2007-2012: arranque parejo en la vida. México DF: Secretaría de Prevención y Promoción de la Salud, Secretaría de Salud de México; 2008.

13. Secretaría de Salud de México. Programa de acción específico 2007-2012: planificación familiar y anticoncepción. México DF: Secretaría de Prevención y Promoción de la Salud, Secretaría de Salud de México; 2008.

14. Secretaría de Salud de México. Programa de acción específico 2007-2012: salud sexual y reproductiva para adolescentes. México DF: Secretaría de Prevención y Promoción de la Salud, Secretaría de Salud de México; 2008.

15. Secretaría de Salud de México. Programa de acción específico 2007-2012: cáncer cervicouterino. México DF: Secretaría de Prevención y Promoción de la Salud, Secretaría de Salud de México; 2008.

16. Secretaría de Salud de México. Programa de acción específico 2007-2012: cáncer de mama. México DF: Secretaría de Prevención y Promoción de la Salud, Secretaría de Salud de México; 2008.
17. Secretaría de Salud de México. Programa de acción específico 2007-2012: prevención y atención de la violencia familiar y de género. México DF Secretaría de Prevención y Promoción de la Salud, Secretaría de Salud de México; 2008.

18. Oona C, Graham W. Strategies for reducing maternal mortality: getting on with what works. Lancet 2006; 368:1284-99.

19. Secretaría de Salud de México. Rendición de cuentas en salud 2010. México DF: Secretaría de Salud de México; 2011.

20. Torres G, Ortega C, Ángeles A, Allen B, Rojas R, Villalobos A, et al. Prevención y diagnóstico temprano de cáncer en la mujer: soluciones al alcance. Salud Pública Méx; en prensa.

21. Instituto Nacional de Estadística y Geografía. Encuesta Nacional de la Dinámica Demográfica 2009. México DF: Instituto Nacional de Estadística y Geografía; 2010.

22. Olaíz G, Rico B, Del Río A. Encuesta Nacional sobre Violencia Contra las Mujeres. México DF: Instituto Nacional de Salud Pública; 2003.

23. Valdéz R, Ruíz M. Violencia doméstica contra las mujeres, ¿cuándo y cómo surge como un problema de salud pública? Salud Pública Méx 2009; 51:505-11.

24. Valdéz R, Mojarro M, Hidalgo EC, Rivera L, Ramos L. Población joven y violencia interpersonal: reducción de la vulnerabilidad y opciones de futuro Salud Pública Méx; en prensa.

25. Knaul F, Nigenda G, Lozano R, Arreola-Ornelas H, Langer A, Frenk J. Brest cancer in Mexico: a pressing priority. Reprod Health Matters 2008; 16:113-23.

26. Jamieson DJ, Honein MA, Rasmussen SA, Williams JL, Swerdlow DL, Biggerstaff MS, et al. H1N1 2009 influenza virus infection during pregnancy in the USA. Lancet 2009; 374:451-58.

27. Torres A. La influenza pandémica $\mathrm{A}(\mathrm{H} 1 \mathrm{~N} 1)$ en mujeres embarazadas. Ginecol Obstet Méx 2010; 78:121-7.

28. Amaya J, Restrepo S. Tamizaje para cáncer de cuello uterino: cómo, desde y hasta cuándo. Rev Colomb Obstet Ginecol 2005; 56:59-67.

29. Hidalgo A. El cáncer cervicouterino, su impacto en México y el por qué no funcional el programa nacional de detección oportuna. Rev Bioméd (México) 2006; 17:81-4

30. Gutiérrez JP. Cobertura efectiva en salud: reforzar la prevención y reducir la brecha socioeconómica. Salud Pública Méx; en prensa.

31. Secretaría de Gobernación. Constitución política de los Estados Unidos Mexicanos. Diario Oficial de la Federación 2012; 15 oct.

32. Ahmed S, Qingfeng L, Lui L, Tsui AO. Maternal deaths averted by contraceptive use: an analysis of 172 countries. Lancet 2012; 380:111-25.

33. Averting Maternal Death and Disability. Averting Maternal Death and Disability program report 1990-2005. New York: Averting Maternal Death and Disability; 2006. 
34. Freedman L, Graham G, Brazier E, Smith JM, Ensor T, Fauveau V, et al. Practical lessons from global safe motherhood initiatives: time for a new focus on implementation. Lancet 2007; 370:1383-91.

35. Presidencia de la República. Convenio general de colaboración interinstitucional para la atención de la emergencia obstétrica, celebrado entre el Instituto Mexicano del Seguro Social, el Instituto de Seguridad y Servicios Sociales de los Trabajadores del Estado y la Secretaría de Salud el 28 de mayo de 2009 por decreto presidencial. http://www.dgpla des.salud.gob.mx/descargas/convenios_emergen cias_obs/ceo_conv.pdf (accedido el 11/Sep/2012).

36. Ramírez G. Integración del Sistema de Salud en México: el caso de la atención de emergencias obstétricas [Disertación de Maestría]. México DF: Instituto Nacional de Salud Pública; 2012.
37. Freyermuth G. Desiguales en la vida, desiguales para morir. La mortalidad materna en Chiapas: un análisis desde la inequidad. New York: Programa de las Naciones Unidas para el Desarrollo, 2010. (Documento de Trabajo 2010/1).

38. Freyermuth G. Los derechos humanos y la salud materna: entre el discurso del siglo XX y las prácticas del XXI. Rev CONAMED 2012; 18:88-95.

39. Gutiérrez JP, Hernández M, Lee GM. Cobertura universal en salud: los retos del monitoreo y la afiliación en jóvenes. Salud Pública Méx; en prensa.

Recibido el 09/Mar/2014

Versión final presentada el 17/Jul/2014

Aprobado el 04/Sep/2014 